\title{
A PROBLEM IN PARTITIONS ${ }^{1}$
}

\author{
MARSHALL HALL
}

Let $m$ objects $x_{1}, \cdots, x_{m}$ be given and from these $n$ non-void subsets $a_{1}, \cdots, a_{n}$ be formed. This partition will determine a matrix $\left(a_{i j}\right)$ in which $a_{i j}=1$ if $a_{i}$ and $a_{j}$ have a non-void intersection and $a_{i j}=0$ if $a_{i}$ and $a_{j}$ are disjoint. Necessarily $\left(a_{i j}\right)$ is a symmetric matrix with 1 's on the main diagonal. The following question has arisen in Ore's investigation of the theory of relations: Is every matrix $\left(a_{i j}\right)$, $i, j=1, \cdots, n$, with $a_{i i}=1, a_{i j}=a_{j i}=0$ or 1 the partition matrix of $n$ objects into $n$ non-void subsets? As will be seen presently, the answer to this question is in the negative. The reason is not that there is any inherent contradiction within certain matrices but that it is not always possible to find a partition of as few as $n$ objects determining a given matrix.

The answer is affirmative for $n=1,2,3,4$ as may be found by direct calculation, but is negative for $n \geqq 5$. It is almost trivial that for $n \geqq 3, m=\left(n^{2}-n\right) / 2$ objects will suffice. Take $\left(n^{2}-n\right) / 2$ objects $u_{i j}=u_{j i}, i \neq j, i, j=1, \cdots, n$, and assign $u_{i j}$ to both $a_{i}$ and $a_{j}$ if $a_{i j}=1$ and discard $u_{i j}$ if $a_{i j}=0$. This will leave certain subsets $a_{i}$ void for which $a_{i i}=1, a_{i j}=0$ if $j \neq i$, and for these we introduce new objects $u_{i}$ in $a_{i}$ alone. If there are one or two such $i$ 's we have discarded at least two $u_{i j}$ 's since $n \geqq 3$. If there are $s \geqq 3$ such $i$ 's we have discarded at least $s(s-1) / 2 u_{i j}$ 's, namely those with both subscripts from this set. In all events we have discarded at least as many objects as we have added and we have a partition of $\left(n^{2}-n\right) / 2$ or fewer objects into $n$ non-void subsets corresponding to the prescribed partition matrix. But it is clear that this number $m=\left(n^{2}-n\right) / 2$ is too high for $n>3$ since the full number of objects is used only if every $a_{i j}=1$ and in this case a single object assigned to every subset will suffice.

Theorem 1. A given matrix $\left(a_{i j}\right), i, j=1, \cdots, n$, in which $a_{i i}=1$, $a_{i j}=a_{j i}=0$ or 1 , is the partition matrix of a set of at most $n$ objects for $n=1,2,3,4$ and of at most $n^{2} / 4$ ( $n$ even and $\left.n \geqq 4\right)$ or $\left(n^{2}-1\right) / 4$ ( $n$ odd and $n \geqq 5$ ).

Proof. Evidently renumbering the subsets makes no difference in the problem. This operation corresponds to permuting both the rows and columns of the matrix, the same permutation being applied to both. Such an operation defines an equivalence on the matrices. The

\footnotetext{
${ }^{1}$ Presented to the Society, February 22, 1941.
} 
non-equivalent matrices of orders 2 and 3 are

$$
\left(\begin{array}{ll}
1 & 0 \\
0 & 1
\end{array}\right), \quad\left(\begin{array}{ll}
1 & 1 \\
1 & 1
\end{array}\right),
$$

$$
\left(\begin{array}{lll}
1 & 0 & 0 \\
0 & 1 & 0 \\
0 & 0 & 1
\end{array}\right), \quad\left(\begin{array}{lll}
1 & 0 & 0 \\
0 & 1 & 1 \\
0 & 1 & 1
\end{array}\right), \quad\left(\begin{array}{lll}
1 & 1 & 0 \\
1 & 1 & 1 \\
0 & 1 & 1
\end{array}\right), \quad\left(\begin{array}{lll}
1 & 1 & 1 \\
1 & 1 & 1 \\
1 & 1 & 1
\end{array}\right) \text {. }
$$

Those of order 2 require 2 and 1 objects respectively and those of order 3 require $3,2,2$, and 1 objects respectively. There are eleven classes of matrices of order four of which only two,

$$
\left(\begin{array}{llll}
1 & 0 & 0 & 0 \\
0 & 1 & 0 & 0 \\
0 & 0 & 1 & 0 \\
0 & 0 & 0 & 1
\end{array}\right), \quad\left(\begin{array}{llll}
1 & 0 & 1 & 1 \\
0 & 1 & 1 & 1 \\
1 & 1 & 1 & 0 \\
1 & 1 & 0 & 1
\end{array}\right)
$$

require as many as four objects. Four classes require three, four classes require two, and one requires one.

Proof for $n=5$. If $a_{i j}=0$ for $j \neq i$, the matrix requires exactly five objects, one in each subset. If some $a_{i j}=1$ renumber to make this $a_{12}$, and suppress the first two rows and columns. The remaining matrix for $a_{3}, a_{4}$, and $a_{5}$ will require at most three objects, and only two unless $a_{34}=a_{35}=a_{45}=0$. Now take four objects $u_{12}, u_{3}, u_{4}, u_{5}$. Assign $u_{i}$, $i=3,4,5$, to $a_{1}$ and $a_{i}$ if $a_{1 i}=1$ and to $a_{2}$ and $a_{i}$ if $a_{2 i}=1$, and discard it if $a_{1 i}=a_{2 i}=0$. If there is an $i$ for which $a_{1 i}=a_{2 i}=1$, then $u_{i}$ is common to both $a_{1}$ and $a_{2}$ and we may discard $u_{12}$. If not assign $u_{12}$ to both $a_{1}$ and $a_{2}$. These four objects and those used for the matrix for $a_{3}, a_{4}, a_{5}$ realize the partition with at most six elements save in the case in which exactly one of $a_{1 i}, a_{2 i}$ is 1 for $i=3,4,5$ and $a_{34}=a_{35}=a_{45}$ $=0$. Now we may discard the elements placed in $a_{3}, a_{4}, a_{5}$ originally and the partition is realized using only the four elements $u_{12}, u_{3}, u_{4}$, and $u_{5}$.

Proof for $n \geqq 6$. If $a_{i j}=0$ for $j \neq i$ we assign a single element $x_{i}$ to each subset $a_{i}$ and realize the partition with exactly. $n$ objects. If some $a_{i j}=1, j \neq i$, renumber so that this is $a_{12}$. Now suppress the first and second rows and columns. We have a partition matrix for $n-2$ subsets which, by an induction hypothesis, may be realized with at most $(n-2)^{2} / 4$ or $\left[(n-2)^{2}-1\right] / 4$ objects, depending on whether $n$ is even or odd. We now use $n-1$ new objects $u_{12}, u_{3}, u_{4}, \cdots, u_{n}$. We assign $u_{12}$ to both $a_{1}$ and $a_{2}$. For $i \geqq 3$ if $a_{1 i}=1, a_{2 i}=0$ we assign $u_{i}$ to $a_{1}$ 
and $a_{i}$. If $a_{1 i}=0, a_{2 i}=1$ we assign $u_{i}$ to $a_{2}$ and $a_{1}$. If $a_{1 i}=a_{2 i}=1$ we assign $u_{i}$ to $a_{1}, a_{2}$, and $a_{i}$. If $a_{1 i}=a_{2 i}=0$ we discard $u_{i}$. This yields a realization of the prescribed partition, using at most $n-1$ more objects. Since $(n-2)^{2} / 4+n-1=n^{2} / 4$ and $\left[(n-2)^{2}-1\right] / 4+n-1$ $=\left(n^{2}-1\right) / 4$, the truth of the theorem for $n-2$ implies the truth for $n$, and the truth of the theorem for $n=4,5$ completes the proof by induction.

We note that the new objects used are never assigned to more than three subsets. For $n=5$ this is also true, and for $n=4$ an object may be assigned to all four subsets only if every $a_{i j}$ is 1 . But here four objects $u_{123}, u_{124}, u_{134}, u_{234}$ assigned respectively to the three appropriate subsets realize the partition. Hence it is never necessary to assign an object to more than three subsets.

THEOREM 2. If $n=2 s$ is even, the symmetric matrix $\left(a_{i j}\right)$ with $a_{i i}=1$; $a_{i j}=0$ for $j \neq i$ and $i, j=1, \cdots, s ; a_{i j}=1$ for $i=1, \cdots, s$, and $j=s+1, \cdots, 2 s ; a_{i j}=0$ for $j>i$ and $i, j=s+1, \cdots, 2 s$, is the partition matrix of $n^{2} / 4$ but no fewer objects. If $n=2 s+1$ is odd, the matrix $\left(a_{i j}\right)$ with $a_{i i}=1, a_{i j}=0$ for $j>i$ and $i, j=1, \cdots, s ; a_{i j}=1$ for $i=1, \cdots, s$ and $j=s+1, \cdots, 2 s+1 ; a_{i j}=0$ for $j>i$ and $i, j=$ $s+1, \cdots, 2 s+1$, is the partition matrix of $\left(n^{2}-1\right) / 4$ but no fewer objects. A partition matrix of order $n \geqq 5$ requiring $n^{2} / 4$ objects ( $n$ even) or $\left(n^{2}-1\right) / 4$ objects ( $n$ odd) is equivalent to the appropriate matrix above.

Proof. In any partition if an object $x$ belongs to as many as three subsets $a_{i}, a_{j}, a_{k}$, then in the partition matrix we have $a_{i j}=a_{i k}=a_{j k}=1$. But in the above matrices $a_{i j}=a_{i k}=1(i, j, k$ different) always imply $a_{j k}=0$. For if $i \leqq s$ and $a_{i j}=a_{i k}=1$, then $j \geqq s+1, k \geqq s+1$ and $a_{j k}=0$; and if $i \geqq s+1, a_{i j}=a_{i k}=1$ then $j \leqq s, k \leqq s$ and $a_{j k}=0$. Hence the matrices above must come from partitions in which no object appears in more than two subsets. Thus in these matrices each 1 above the main diagonal corresponds to at least one object in common to two subsets and these objects must all be different. Hence these are the partition matrices of $n^{2} / 4$ or $\left(n^{2}-1\right) / 4$ objects but no fewer.

Consider a matrix $\left(a_{i j}\right), n \geqq 5$, requiring the maximum number of objects. If some $a_{i i}=1$ and all $a_{i j}=0, j \neq i$, then we may adjoin a single element for $a_{i}$ to a partition for the remaining $n-1$ subsets and have a partition with less than the maximum number of objects. First suppose $n \geqq 6$. In the proof of Theorem 1 for $n \geqq 6$ note that if $a_{1 i}=a_{2 i}=0$ we discard $u_{i}$ and have fewer than $n-1$ new objects to adjoin. Also if $a_{1 i}=a_{2 i}=1$ we may discard $u_{12}$ since $u_{i}$ is an object common to both $a_{1}$ and $a_{2}$. In these cases the partition requires fewer 
than $n^{2} / 4$ or $\left(n^{2}-1\right) / 4$ objects. Since, by renumbering, any triple $i, j, k$ may be taken into $1,2, i$, it follows that in a matrix requiring the maximum number of objects exactly two of $a_{i j}, a_{i k}, a_{j k}$ must be 1's and the third must be 0 or all three must be 0 . For a nonvanishing triple renumber so that the common subscript of the two 1's is 1 and so that the first row is of the form $a_{11}=1, a_{1 i}=0, i=2, \cdots, t, a_{1 i}=1$, $i=t+1, \cdots, n$. Here $t \leqq n-2$ since there are at least two 1 's in this row. Now $a_{i j}=0$ if $j \neq i, i, j \geqq t+1$, since otherwise $a_{1 i}, a_{1 j}, a_{i j}$ would all be 1 's. Also $a_{i j}=1$ if $i \leqq t, j \geqq t+1$, since $a_{1 i}=0, a_{1 j}=1$. Finally $a_{i j}=0$ if $j \neq i$ and $i, j \leqq t$. For $a_{n i}=1, a_{n j}=1$ by the preceding argument. This completely determines the matrix which has a rectangle containing $t(n-t) 1$ 's in the upper right-hand corner and also in the lower left-hand corner but 0 's elsewhere apart from the main diagonal. By placing objects $u_{i j}$ in $a_{i}$ and $a_{j}$ if $a_{i j}=1, j \neq i$, we may realize this partition using $t(n-t)$ objects. These objects are certainly different since none may be in three subsets. If $n=2 s$ is even $t(n-t)<n^{2} / 4$ unless $t=s$ which yields the matrix of the theorem. If $n=2 s+1, t(n-t)$ $<\left(n^{2}-1\right) / 4$ unless $t=s$ or $s+1 . t=s$ yields the matrix of the theorem, while $t=s+1$ yields an equivalent matrix. The permutation $(1, n)(2, n-1) \cdots(s, s+1)$ of the rows and columns interchanges these two matrices.

Finally suppose $n=5$. Here $\left(n^{2}-1\right) / 4=6$. Suppose for some triple, say $1,2,3, a_{12}=a_{13}=a_{23}=1$. Then as in the proof of Theorem 1 for $n=5$, to a partition of objects for $a_{3}, a_{4}, a_{5}$ we need add at most three objects. If these three subsets required only two objects, we have used at most five objects for the entire partition. If they required three objects, then $a_{34}=a_{35}=a_{45}=0$ and we may discard the object originally placed in $a_{3}$ and have a complete partition using at most five objects. Hence there is no triple $i, j, k$ for which $a_{i j}=a_{i k}=a_{j k}=1$. Similarly if for some triple, say $1,2,3, a_{12}=1, a_{13}=0, a_{23}=0$ we need to add at most three objects to the partition for $a_{3}, a_{4}, a_{5}$ and if this requires three objects then either someone of the old objects may be discarded or one of the new objects is discarded. Hence if $a_{12}=1$, one of $a_{13}, a_{23}$ is 1 and the other 0 . From here the proof as for $n \geqq 6$ applies.

YALE UNIVERSITY 